\title{
Study of the correlation of factors affecting frequency of social activity transfer from virtual environment to real-world environment and vice versa
}

\author{
Anton Zagranichniy* \\ National Research Saratov State University named after N. G. Chernyshevskiy, 410012, \\ Saratov, Russia
}

\begin{abstract}
The article presents research results demonstrating interrelation between factors affecting frequency of social activity transfer from the virtual environment to the real-world one and vice versa. In the course of the study we investigated 19 different factors, including sociodemographic characteristics, amount and specificity of contacts in each of the environments, subjective assessment of social activity characteristics in various environments. The study involved 214 respondents aged 15 to 24 from the cities of Balakovo, Saratov, and Moscow. We analysed and interpreted the correlation interrelation via social activity transfer from one environment to another and by such factors as: place of residence size; number of friends in the virtual environment; factor regarding frequency of misconduct situations that were followed by moral responsibility in the real environment; factor of compliance with social norms in the virtual environment.
\end{abstract}

Modern scientific research has increasingly become interdisciplinary, and covers several subject-areas within a specific scientific subject. It has become clear that studying certain phenomena is more productive from the point of view of their correlation and interaction with other phenomena. Analysis of this approach is presented in the academic papers by V. A. Mazilov. In these works he comes to the conclusion that interdisciplinary and comprehensive research is seen as the most effective solution to fundamental problems which psychological research faces nowadays [1]. Such approach of complex researches is used, in psychological science rather volume, for example, in the course of studying of public work Shamionov R.M. points, that public activity and, as a result, public transformations are the cross-disciplinary problem demanding the corresponding approach to studying [2]. Since it has been established that personality and environment are connected via mutual influence [3], a perspective way to study the interaction between personality and environment is the integrated approach that allows us to study not only the phenomena of specific systems (i.e. personality and environment), but the process of interaction between these systems as well.

\footnotetext{
*Corresponding author: zagr.93@inbox.ru
} 
A particularly valuable object of study in this area is social activity of an individual. In works written by Shamionov R. M., Grigoryeva M. V., Grigoryeva A. V. the authors come to the conclusion that social activity is not only a personal activity act, but also an act of environment transformation. In the same work, they give a fairly accurate definition of social activity: social activity of individuals and groups involves not only participation in public life, but, above all, initiative-based and creative attitude to the spheres of social environments, as well as to oneself as a subject of social being [4]. The importance of studying social activity study is emphasized in works of various psychologists and researchers. Thus, in the paper devoted to social activity of youth and approaches to assessing the forms, motives and factors of social activity manifestation in the modern Russian society, Trotsuk I. V. and Sokhadze K. G. talk about the guiding motives of subjective "measurement", structural levels, types of goals, as well as the implementation forms, external and internal factors of development. The authors come to the conclusion that studying social activity is relevant and requires more in-depth research from different perspectives [5]. Kudinov S. S. suggests that social activity contributes to a more fruitful socialization and self-realization of a person; he views social activity as the basis of personal self-realization [6]. In the work entitled "Social activity of an individual and a group: definition, structure, mechanisms" Shamionov R. M. emphasizes the importance of social activity study from the perspective of unlocking the potential for the development of an individual, group, and society [7]. Arguing on social activity in terms of subjective ideas of the personality of socially active person, Shamionov R.M. and Grigoriev A.V. come that the image of socially active person is connected with emotionally positive background, cheerfulness and spontaneity and such subjective categories as an initiative and confidence [8]. That is have ideas of socially active person the general positive background and is connected with development of productive subjective characteristics of the personality. Development idea of itself as about socially active personality, first of all through participation in social activity, promotes increase in subjective wellbeing of the personality and can be useful in terms of development of significant subjective characteristics that indicates practical benefits of a research of social activity.

However, most of the works on social activity are within the classical paradigm of the real-world environment. Today it has become clear that this area of scientific knowledge has to be expanded, since the influence and functions of the virtual environment, which has its own specifics, are expanding. This environmental specificity forms a number of features in the social activity manifestation in the virtual environment. Scientific works by Panich O. E., Ovsyannikova E. A., and Khudaeva M. Yu. are devoted to peculiarities of virtual environment influence on teenage personality [9], as well as the paper written by Yelnikova O. E., which deals with features of socialization of modern students in terms of virtual educational environment impact [10], prove the fact that the volume and functions of virtual environment are expanding. The authors unanimously record the significance of virtual environment's influence on the basic processes of personal formation, such as socialization and adaptation. Therefore, we can say that nowadays individual social activity study must necessarily include a study of environmental characteristics of not only the classical paradigm of the real-world environment, but also of the relatively new virtual environment.

Before moving on to the study of social activity in the virtual environment, we need to understand the correlation of environments within the act of activity and to study the mechanism of social activity transfer from one environment to another. The most perspective social group for this task is the "youth". Since this social group does not only give us the widest access to the study of activity in the virtual environment, it also allows us to consider the prospects for the development of the phenomenon under study. 
This study involved 214 respondents from the cities of Balakovo, Saratov, and Moscow. The age of respondents ranged from 15 to 24 .

The research process involved a questionnaire, as a result of which we clarified the socio-demographic data of the respondents, evaluated the features of the real-world and virtual environment and interaction processes for various types of social activity within these environments, described the mechanism of activity transfer from one environment to another and vice versa by various criteria.

This work is particularly focused on the correlation of factors affecting the frequency of social activity transfer from one environment to another. The purpose of this study is to attempt to comprehend the element of social activity transfer mechanism from one environment to another.

In the course of the study we investigated 19 different factors, including sociodemographic characteristics, the amount and specificity of contacts in each of the environments, subjective assessment of the social activity characteristics in various environments. Speaking of factors affecting the frequency of social activity transfer from one environment to another, we have the following significant correlation interrelations. The size of the place of residence has a direct significant correlation with the frequency of social activity transfer from virtual environment to the real-world one $(r=0.224 ; p<0.01)$. That is, the larger the place of residence is, the more often activity is transferred from virtual environment to the real-world one. Residents of big cities transfer social activity from the virtual environment to the real-world one more often than residents of small towns or villages. This can be explained by the quantity and quality of socio-cultural process acts' organization. In large cities social and cultural life is more developed, it creates more opportunities for various forms of social activity for an individual, and, therefore, increases the frequency of social activity transfer. In addition, nowadays activity in the virtual environment often precedes in the process of organizing social activity in the real environment. This explains the frequency of social activity transfer from the virtual environment to the real-world one. Moreover, the number of friends in the virtual environment factor $(\mathrm{r}=0.279 ; \mathrm{p}<0.01)$ has a direct significant correlation with the frequency of social activity transfer from the virtual environment to the real-world one. It should be noted that the same correlation exists with the reverse transfer from the realworld environment to the virtual environment $(\mathrm{r}=0.332 ; \mathrm{p}<0.01)$. Therefore, the more friends a person has in any of the environments, the more often the activity will be transferred from one environment to another, which can be explained by the number of social contacts. The more social contacts a person has in each environment, the greater the likelihood that these social contacts are available both in the real-world environment and in the virtual environment. Obviously, there are situations when a particular person has a larger number of contacts in one of the environments and contacts can be isolated in one of the environments. But thinking about the general dynamics, we can talk about the proportional distribution of contacts and their duplication in different environments. Consequently, a large amount of social contacts in each of the environments contributes to social activity transfer from one environment to another. The factor related to frequency of misconduct situations, followed by moral responsibility in the real environment, has a direct significant correlation with the frequency of social activity transfer from the virtual environment to the real-world one. $(r=0.267 ; p<0.01)$. In this case, the frequency of misconducts, followed by moral responsibility in the real-world environment, may be a consequence of the frequent activity transfer from the virtual environment to the real-world one. Since frequent activity transfer from the virtual environment to the real-world one implies the continuation of an act of activity in the real-world environment, it becomes clear that the activity process involves, among other things, the commission of such acts. Otherwise, we are talking about the fact that this correlation indicates general assessment of 
actions followed by moral responsibility, and we can assume that in the virtual environment social norms are observed to a lesser extent or are interpreted differently. Probably, a person, in some cases, evaluates his/her moral responsibility differently, committing the same deed followed by moral responsibility in different environments. This assumption is supported by the established direct significant correlation between factors: compliance with social norms in the virtual environment and social activity transfer from one environment to another. The more often respondents believe that the norms in the virtual environment are observed to a lesser extent than in the real-world one, the more activity is transferred from the virtual environment to the real-world environment $(\mathrm{r}=0.188 ; \mathrm{p}<0.05)$. The distinguished connection is quite logical, since it is obvious that if respondents believe that social norms are less respected in the virtual environment, this creates discomfort and reduces the person's desire to create acts of activity under such conditions, which leads to transfer of the act to another, more comfortable environment. However, it has also been established that the more often respondents consider that the norms in the virtual environment are observed less than in the real-world one, the more activity is transferred from the real-world environment to the virtual environment $(r=0.191 ; p<0.05)$. This contradicts the logic of the previously established correlation and can be explained either by the fact that a person consciously joins an activity in the virtual environment, assuming a lower level of compliance with social norms in the process of interaction, which tells us about the significant advantages of the virtual environment for some types of activity, which explain the reduced requirements for compliance with social norms, or such a correlation is a consequence of activity in the virtual environment. Respondents initially transferred activity to the virtual environment and only then, in the process of activity, came to the conclusion that social norms in the virtual environment were observed to a lesser extent. However, since respondents have repeated, varied experiences of activity in the virtual environment, we can assume that the first hypothesis on this correlation is more likely. One way or another, the presence of such correlations tells us that a person, despite the reduced moral requirements in the virtual environment, is ready for activity within this environment.

Summarizing, we can say that in the process of studying various factors affecting social activity transfer from one environment to another, we identified the following significant correlations: place of residence size is correlated with activity transfer from the virtual environment to the real-world environment; the total number of friends is correlated with mutual social activity transfer from one environment to the other. The interrelation within these factors looks quite logical and consistent. Therefore, the greatest interest for us is the correlation between the activity transfer from the virtual environment to the real-world environment and the frequency of actions, followed by moral responsibility in the realworld environment, as well as the correlation between assessing the level of compliance with social norms and activity transfer from one environment to another and vice versa. We see that respondents are ready for activity in the virtual environment, even if this activity is associated with lower level of compliance with social norms. In the process of analysing subjective assessments of respondents, we see that respondents often do things that are followed by moral responsibility in the real world. However, this probably does not signify quantitative indicator of such actions, but rather indicates different assessment of such acts in different environments. Since we have also established that respondents transfer social activity from the real-world environment to the virtual environment, even if they understand that social norms in the virtual environment are observed to a lesser extent, this may indicate reduced moral assessment of interactions in relation to the real-world environment. This also indicates the value of activity characteristics in the virtual environment, which compensates for the reduced moral requirements for the act of activity. We can assume that one of the main environmental features of the virtual environment is a 
reduced level of requirements for social norms of interaction, which is probably compensated by the exceptional value of activity acts characteristics for certain types of activities in the virtual environment.

The study was supported with a grant from the Russian Science Foundation (project No. 18-1800298)

\section{References}

1. V. Mazilov, Yaroslavl Pedagogical Bulletin. 4, 201-211 (2013)

2. R. Shamionov. ICERI2018 Proceedings, 11th International Conference of Education, Research and Innovation. November 12th-14th. Seville, Spain; 95439548 (2018)

3. M. Grigorieva, The World of Psychology. 1, 93-101 (2008)

4. R. Shamionov, M. Grigoryeva, A. Grigoryev, Social Psychology and Society. 1, 18-34 (2019)

5. I. Trotsuk, K. Sokhadze. Bulletin of the RUDN University. Series: Sociology. 4, 58-74 (2014)

6. S. Kudinov. Acmeology. S1-2, 124-125 (2014)

7. R. Shamionov. Bulletin of the RUDN University. Series: Psychology and Pedagogy. 4, 379-394 (2018)

8. R. Shamionov, A. Grigoryev, International Journal of Cognitive Research in Science Engineering and Education (IJCRSEE). 7(1), 15-20 (2019)

9. O. Panich, E. Ovsyannikova, M. Khudaev, Problems of Modern Teacher Education. 55-9, 349-355 (2017)

10. O. Elnikova, The Psychology of Education in a Multicultural Space. 1-21, 10-15 (2013) 